\section{AN OPEN LABEL PHASE IA/IB STUDY FOR SAFETY, PHARMACOKINETICS (PK), AND EFFICACY OF ONC 392 AS A SINGLE AGENT AND IN COMBINATION WITH PEMBROLIZUMAB IN ADVANCED SOLID TUMORS}

${ }^{1}$ Pan Zheng ${ }^{*},{ }^{2}$ Kun He, ${ }^{1}$ Yang Liu. ${ }^{1}$ OncoC4, Inc., Rockville, MD, USA; ${ }^{2} R$ and G Pharma US, Inc., Rockville, MD, USA

Background ONC-392 is a highly selective, humanized monoclonal IgG1 antibody against CTLA-4. The parental clone was identified through in vivo screening in a humanized CTLA-4 mouse model for high anti-tumor efficacy and low autoimmune toxicity. By preserving CTLA-4 on the cell surface, ONC 392 leaves a higher ligand density for better antibodydependent cellular cytotoxicity (ADCC), resulting in more efficient in Treg depletion in the tumor microenvironment (TME) and more potent tumor rejection in pre-clinical models. Based on encouraging Phase I dose escalation study, a major revision of the protocol has been performed to expand clinical indications among patients with advanced solid tumors.

Methods This is a Phase IA/IB, open label, dose-escalation, and dose-expansion study of intravenous (IV) ONC 392 as a single agent and in combination with Pembrolizumab (anti PD-1, marketed as KEYTRUDA ${ }^{\circledR}$ by Merck) in patients with advanced/metastatic solid tumors. The study consists of three parts: (1) Part A (Figure 1) is a dose-finding rapid titration study of ONC-392 as a single agent in patients with advanced solid tumors of various histology to define the recommended Phase II dose for ONC-392 monotherapy (RP2D-M). (2) Part B (Figure 2 and 3) has Part B1 and Part B2 as dose-finding for combination therapy with either pembrolizumab or Osimertinib $80 \mathrm{mg}$ orally once daily to define the recommended Phase II dose for ONC-392 in combination with either drug (3) Part C (Figure 4) Phase IB expansion cohorts of ONC392 in monotherapy and in combination therapy with Pembrolizumab to determine safety and initial efficacy. A total of 8 cohorts encompassing monotherapy for pancreatic cancer and triple negative breast cancer and combination therapy of non-small cell lung carcinoma, melanoma and Markel cell carcinoma. The primary endpoints for Part A and B are safety and tolerability to observe maximal tolerable dose and recommended doses for Phase II, while that for part C is efficacy as measured by overall response rates. The planned enrollment is 300 patients and the study duration is 18 months.

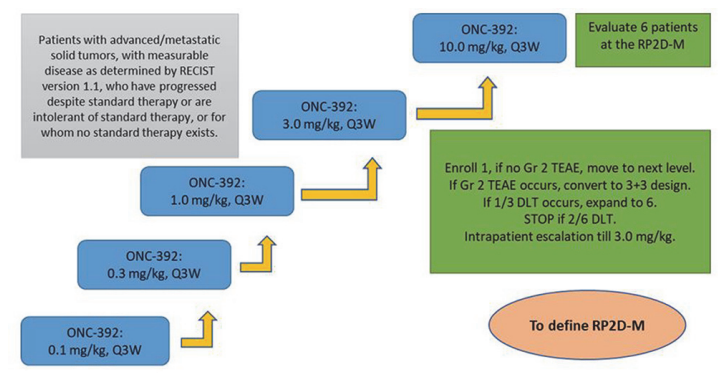

DLT=dose-limiting toxicity; Gl=Grade; Q3W=every 3 weeks; RECIST=Response Evaluation Criteria in Solid Tumors; RP2D-M=recommended Phase II dose for ONC-392 as monotherapy; TEAE=treatment-emergent adverse

Abstract 533 Figure 1 Diagram of ONC-392-001 study Part A

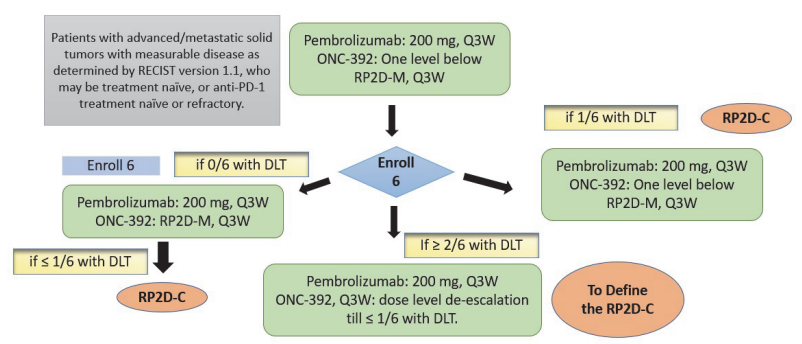

Abstract 533 Figure 2 Diagram of ONC-392-001 study Part B1

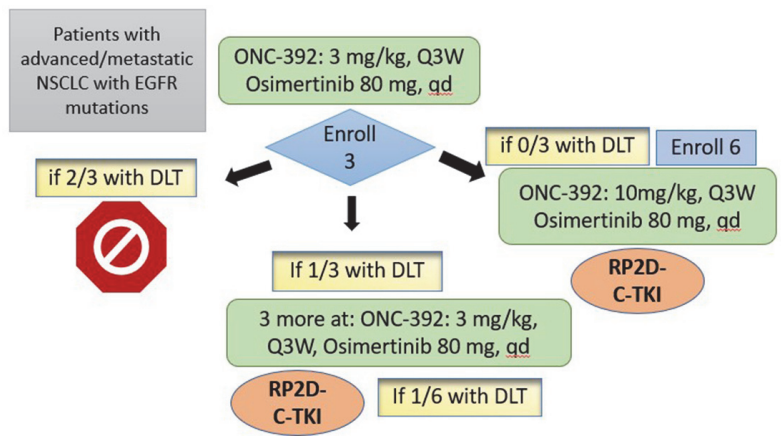

Abstract 533 Figure 3 Diagram of ONC-392-001 study Part B2

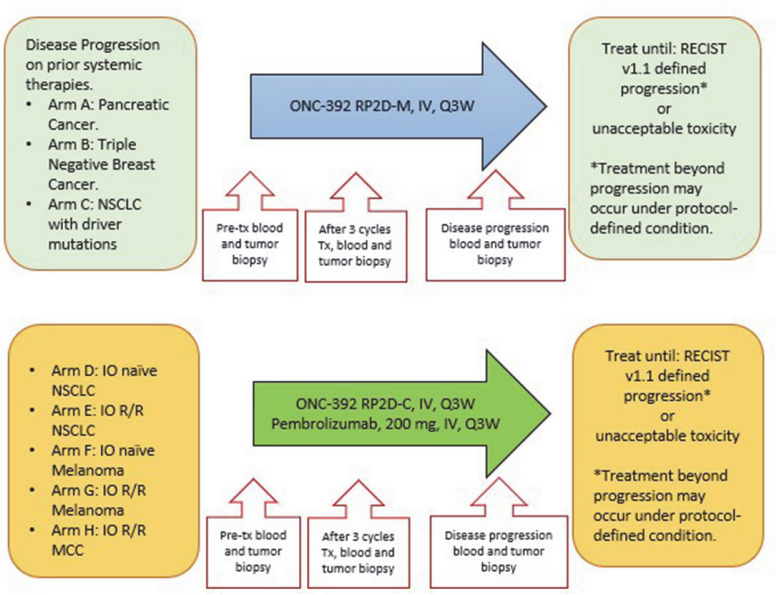

Abstract 533 Figure 4 Diagram of ONC-392-001 study Part C

Acknowledgements The study is partially supported by an NIH grant R44CA250824 to OncoC4, Inc.

Trial Registration NCT04140526

Ethics Approval The study obtained ethics approval from WIRB, study number 20193108. The participants gave informed consent before the enrollment and treatment.

http://dx.doi.org/10.1136/jitc-2021-SITC2021.533 